\title{
Osteocytes and Bone Lining Cells: Which are the Best Candidates for Mechano-Sensors in Cancellous Bone?
}

\author{
M. G. MULLENDER and R. HUISKES
}

Biomechanics Section, Institute of Orthopaedics, University of Nijmegen, Nijmegen, The Netherlands

\begin{abstract}
Previously, we have investigated the possible role of osteocytes as mechano-sensors, and mediators of bone turnover. It was found that the proposed regulatory mechanism produced morphologies of trabecular bone, under particular loading conditions, which were consistent with morphogenesis and adaptation as seen in reality. The main objective of this study was to discern whether lining cells or osteoblasts could possibly play a similar role as effectively with regard to their capacity for self-optimization of the trabecular architecture, in terms of a low apparent mass to stiffness ratio. For that purpose the earlier analyses with osteocytes as mechanosensors, distributed throughout the bone, were repeated for mechano-sensors located at bone surfaces only. Compared to the osteocyte model, the surface cell remodeling algorithm was reluctant to change its architecture, which implies that it is less sensitive to changes in the loading pattern. This resulted in less efficient bone adaptation, which was reflected by a considerably higher relative mass for a similar apparent stiffness in the loading direction. In other words, more mass is needed to obtain an equally stiff structure, at the apparent level, with respect to the externally applied loads. Furthermore, stresses and strains at the tissue level vary across a much wider range, relative to the osteocyte model, where the higher incidence of elevated strains indicates an increased failure risk. Therefore, we conclude that mechanical information at the bone surface may not be sufficient to adequately regulate functional bone adaptation. (Bone 20:527-532; 1997) (C) 1997 by Elsevier Science Inc. All rights reserved.
\end{abstract}

Key Words: Trabecular bone; Bone remodeling; Mechano-sensory system; Osteocyte; Bone lining cell; Computer simulation.

\section{Introduction}

Although it is generally accepted that bone tissue adapts to mechanical demands, the regulatory mechanisms responsible for this process are not understood. For one thing, the mechanism by which the bone tissue senses mechanical stimuli has not been established. Neither the cells responsible for transduction of mechanical into chemical signals nor the means by which the actual bone remodeling processes are subsequently regulated are identified.

Osteocytes, located within the bone matrix, bone lining cells,

Address for correspondence and reprints: R. Huiskes, Biomechanics Section, Institute of Orthopaedics, University of Nijmegen, P.O. Box 9101, 6500 HB Nijmegen, The Netherlands. E-mail: rik@orthp.azn.nl covering the bone surface, and osteoblasts have all been proposed to play important roles as mechano-sensors in the regulatory process. In vitro studies showed that the activity of both lining cells and osteocytes increased after loading. ${ }^{4,18}$ Osteocytes were suggested as the most suitable candidates for the role of mechano-receptors, because of their location and the interconnections by which they communicate with each other and with cells at the bone surface. ${ }^{2,9,11}$ It was proposed that these cells are stimulated by fluid flow in the caniculi, due to mechanical loading of the tissue. ${ }^{6,24}$ In fact, it was shown experimentally that osteocytes are very sensitive to fluid flow across their cell membranes. ${ }^{8}$

The hypothesis that osteocytes sense mechanical signals and regulate the local adaptation of bone mass was recently investigated for its feasibility, using a computer simulation model. ${ }^{14}$ In this regulatory model, bone density was adapted at any location within the tissue, according to a stimulus received from the osteocytes in the vicinity. It was shown that the proposed regulatory mechanism could explain the genesis and adaptation of trabecular patterns in accordance with the external loads, indicating that such a hypothesis is realistic. ${ }^{13,14,20,22}$ Nevertheless, in reality, trabecular bone turnover occurs only at surfaces and not within the tissue. Furthermore, the question remains, if sensors located at the bone surface (lining cells and osteoblasts) could regulate bone remodeling equally well. It was shown in many studies that osteoblasts and osteoblast-like cells are very sensitive to mechanical loads as well (see Burger and Veldhuijzen $^{1}$ for a review). In addition, Miller et al. ${ }^{12}$ and Parfitt ${ }^{17}$ suggested that bone lining cells probably play important roles in bone remodeling by mediating the activation of the bone remodeling sequence.

We have investigated two questions in this article. First, do the results of the regulatory model based on osteocytes as mechano-sensors differ from those previously described, if, as in reality, remodeling is allowed only at trabecular surfaces? Second, can a simulation model, based on the assumption that lining cells and osteoblasts are mechano-sensors and regulators of bone turnover, explain mechanical adaptation of trabecular bone equally well? To investigate the latter question, a model was developed based on the alternative hypothesis that sensor cells are located only on the trabecular surfaces. The behavior of this regulatory model was compared to the regulatory models in which the sensors are located within the bone matrix.

\section{Methods}

Two hypothetical regulatory mechanisms for trabecular bone (re)modeling were compared. In both regulation schemes, local mechanical signals are appraised by sensor cells which subse- 


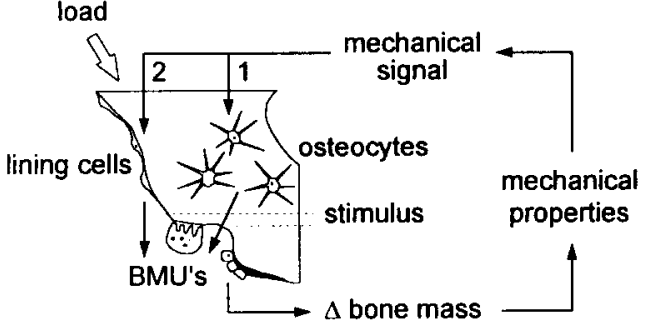

Figure 1. Two alternative hypothetical regulatory schemes are compared. Bone remodeling is assumed to be controlled by local feedback. The first hypothesis is that osteocytes appraise mechanical signals and stimulate BMUs to adapt bone mass (1). The second hypothesis states that bone surface cells (lining cells and osteoblasts) are the mechanosensors and that these cells stimulate bone turnover by BMUs (2). In both schemes, this results in a change of local mechanical properties, which again affects local mechanical signals.

quently stimulate populations of osteoclasts and osteoblasts (basic multicellular units, BMUs) to adapt the local bone mass (Figure 1). In the first regulation model, osteocytes are assumed to be the sensor cells (osteocyte model), while in the second regulatory mechanism it is presumed that only cells covering the trabecular surface act as sensors (surface cell model). For the osteocyte model two possibilities were compared. The first is "overall remodeling," which implies that bone density can be adapted at any location in the tissue. This model was described earlier by Mullender et al. ${ }^{13}$ and Mullender and Huiskes. ${ }^{14}$ The second is "surface remodeling," where the bone density is allowed to change only at the bone-marrow interface. In the surface cell model, surface remodeling was investigated only.

\section{Mathematical Formulation of the Remodeling Hypotheses}

The mathematical foundation of the models was analogous to Mullender and Huiskes. ${ }^{14}$ The strain energy density (SED) was taken as the mechanical signal $S_{i}(t)(\mathrm{MPa}$ ) measured by a sensor cell $i$. The strain energy density is in fact the local elastic (strain) energy stored per volume of bone tissue. According to the difference between the actual signal and a reference signal $k$, the sensor produces a stimulus. The amount of stimulus received by the BMUs depends on the distance between the sensor cell $i$ and the location $x$ of the actor cells. The local stimulus value $F(x, t)$ at location $x$ at time $t$ is the sum of the stimuli received from all sensors:

$$
F(x, t)=\sum_{i=1}^{N} f_{i}(x)\left[S_{i}(t)-k\right]
$$

with $N$ the number of sensors and the spatial influence function

$$
f_{i}(x)=\exp ^{-D / d_{i}(x)},
$$

describing the decrease in stimulus with increasing distance $d_{i}(x)$ ( $\mathrm{mm}$ ) between location $x$ and sensor $i$. The parameter $D$ determines the decay of the influence function.

The change in the relative density $m(x, t)$ in location $x$ is governed by the local stimulus value $F(x, t)$. Hence, in case of overall remodeling

$$
\frac{d m(x, t)}{d t}=\tau F(x, t) \text { with } 0<m(x, t) \leq 1,
$$

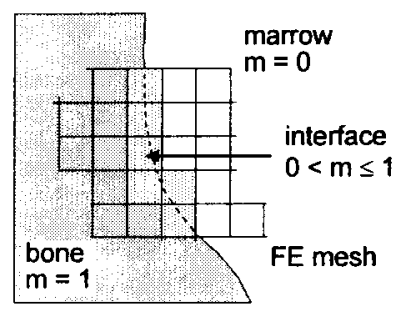

Figure 2. The jagged boundaries of the elements prevent an accurate representation of a smooth bone surface. This problem has been solved by allowing boundary elements to have intermediate densities, representing partial volumes of bone.

and in case of surface remodeling

$$
\begin{cases}\frac{d m(x, t)}{d t}=\tau F(x, t), & x \in \text { trabecular surface } \\ \frac{d m(x, t)}{d t}=0, & x \notin \text { trabecular surface }\end{cases}
$$

$$
\text { with } 0<m(x, t) \leq 1 \text {, }
$$

where $\tau\left(\mathrm{MPa}^{-1} \mathrm{~s}^{-1}\right)$ is a constant regulating the rate of the process. The local elastic properties were calculated from the local relative density using a cubic power relationship in accordance with experimental data. ${ }^{3}$ Hence, the elastic modulus at location $(x)$ was calculated from

$$
E(x, t)=C m^{3}(x, t),
$$

with $C(\mathrm{MPa})$ a constant.

\section{Numerical Formulation}

The regulatory models were applied to a volume of bone tissue. Input to the regulation models was given by the magnitudes and directions of the mechanical loads. In turn, the model predicted the distribution of bone mass in time, i.e., the development of architecture, for given parameter values. The bone tissue was modeled by finite elements (FE), which allowed the calculation of the mechanical variables inside the tissue for externally applied loads. The development of bone architecture in time was simulated numerically, i.e., equations (3) and (4) were solved recursively, using a numerical integration technique (forward Euler) to find the new values for the relative density per element. The procedure was continued until the changes in architecture were virtually zero. A variable time step was used that was calculated from the maximal stimulus and a prescribed maximal change in relative density at any location according to van Rietbergen et al. ${ }^{22}$

For surface remodeling, it was necessary to define a bonemarrow interface within the finite element model as changes in density are only allowed at this location. Bone surfaces were modeled by elements of intermediate density, representing partial volumes of bone and marrow. These elements are located between elements with minimal density (marrow) and elements with maximal density (bone) (Figure 2).

The difference between the osteocyte model and the surface cell model was the location of the sensors. In the osteocyte model, the sensors were uniformly distributed over the mineralized bone matrix. In the surface cell model, the sensors were located in the centers of the elements representing the bone 
Osteocyte model - overall remodeling

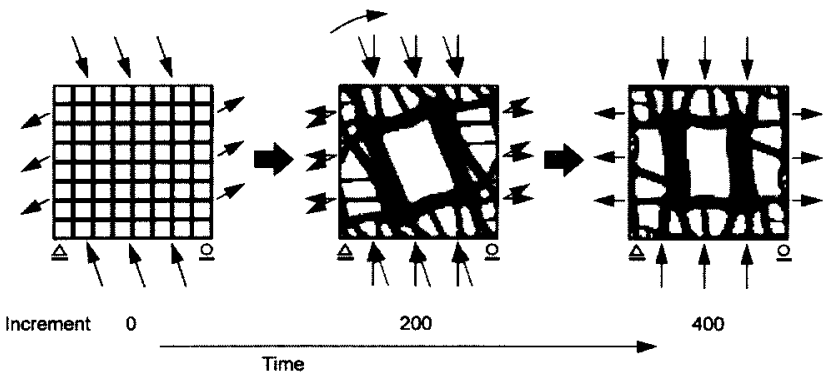

Osteocyte model - external remodeling

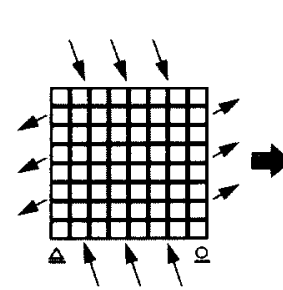

Increment

0

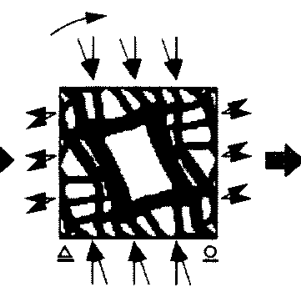

200

Time

Lining cell model

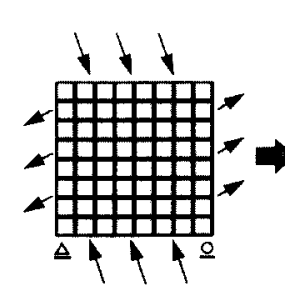

Increment 0

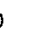

Time

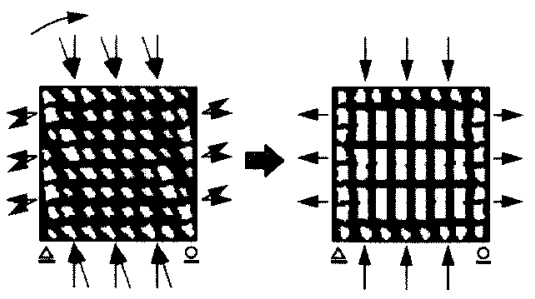

400
Figure 3. The equilibrium architectures are presented for the osteocyte models using overall remodeling (a), and surface remodeling (b), and for the surface cell model (c). The architectures produced by both osteocyte models show large resemblance. Note that the architectures produced by the surface cell model are closer to the initial architecture.

surface. Stresses and strains in the locations of the sensors were calculated via extrapolation of the values in the integration points to the nodal points of each element, and subsequently linear interpolation to the location in which the sensor was situated. The mechanical signal per sensor was calculated according to

$$
S_{i}=\frac{1}{2} \sigma_{i}: \varepsilon_{i}
$$

where $\sigma_{i}$ and $\varepsilon_{i}$ are the stress and strain tensors in the location of sensor $i$.

\section{Application of the Remodeling Hypotheses}

The regulation models were applied to a plate of $2 \times 2 \mathrm{~mm}$ (thickness $0.02 \mathrm{~mm}$ ), meshed with $80 \times 80$ four-node elements. The initial architecture was an arbitrary trabecular structure resembling a lattice (Figure 3). The physiological parameters in
Table 1. Apparent properties of the produced architectures for eachmodel

\begin{tabular}{|c|c|c|c|c|c|}
\hline \multicolumn{2}{|l|}{ Model } & \multicolumn{4}{|c|}{ Apparent properties } \\
\hline \multirow[b]{2}{*}{ Algorithm } & \multirow[b]{2}{*}{ Load case } & \multirow[b]{2}{*}{$\begin{array}{l}\text { Relative } \\
\text { density }\end{array}$} & \multicolumn{3}{|c|}{$\begin{array}{l}\text { Principal Young's } \\
\text { moduli in orthotropic } \\
\text { directions }\end{array}$} \\
\hline & & & $\begin{array}{c}\mathrm{E}_{11} \\
(\mathrm{GPa})\end{array}$ & $\underset{(\mathrm{GPa})}{\mathrm{E}_{22}}$ & $\begin{array}{c}\mathrm{G}_{12} \\
(\mathrm{GPa})\end{array}$ \\
\hline \multicolumn{6}{|l|}{ Osteocyte model } \\
\hline \multirow[t]{2}{*}{ Overall remodeling } & 1 & 0.52 & 1.79 & 1.04 & 0.23 \\
\hline & 2 & 0.52 & 1.85 & 1.08 & 0.17 \\
\hline \multicolumn{6}{|l|}{ Osteocyte model } \\
\hline \multirow[t]{2}{*}{ Surface remodeling } & 1 & 0.55 & 1.89 & 1.03 & 0.22 \\
\hline & 2 & 0.54 & 2.00 & 1.05 & 0.23 \\
\hline \multirow[t]{2}{*}{ Surface cell model } & 1 & 0.61 & $1.92^{\prime}$ & $1.50^{\mathrm{a}}$ & $\mathrm{b}$ \\
\hline & 2 & 0.50 & 1.77 & 1.07 & 0.12 \\
\hline
\end{tabular}

aYoung's modulus in direction of applied load.

bArchitecture was not orthotropic.

the models were the reference energy $k$, the sensor density $n$ [equal to their number $N$ divided by the area of bone tissue (osteocyte model) or divided by the length of the bone perimeter (surface cell model)], the exponential osteocyte-influence function (characterized by the distance parameter $D$ ), and the constants $\tau$ and $C$ (equal to the maximal elastic modulus). $n$ was taken as $1600 \mathrm{~mm}^{-2}$ in the osteocyte model and $40 \mathrm{~mm}^{-1}$ in the surface cell model. Osteocyte density was chosen within a physiological order of magnitude, which was estimated form measurements by Marotti et al. ${ }^{11}$ and Mullender et al. ${ }^{15,16}$ The value for lining cell density was based on an average length of the cells of $25 \mu \mathrm{m}$. However, no actual data of lining cell density were available. A value of $0.02 \mathrm{MPa}$ was used for $k$ and $D$ was $100 \mu \mathrm{m} .{ }^{14} C$ was taken as $6 \mathrm{GPa}^{21}$ and the rate constant was arbitrarily set at $\tau=1 \mathrm{MPa}^{-1} \mathrm{~s}^{-1}$.

The plate was loaded at each side with uniform stress distributions. The stress magnitudes $\sigma_{1}$ and $\sigma_{2}$ were 5 and $-2.5 \mathrm{MPa}$, respectively. After 200 increments (after which stable configurations were reached), the loading configuration was altered by changing the applied stress orientations from $20^{\circ}$ to $0^{\circ}$ relative to the plate. Hereafter, the simulations were continued for another 200 increments.

\section{Evaluation of the Results}

The resulting architectures were evaluated for their apparent mechanical properties and the relative apparent density. To assess the mechanical properties of the architectures produced by the models, the global stiffness matrices were determined for each equilibrium architecture from the structure morphology and the element stiffness matrices according to van Rietbergen et al. ${ }^{23}$ From the global stiffness matrix, the axes of orthotropy and the principal Young's moduli associated with these axes were determined. ${ }^{23}$ To appraise the differences between architectures at the tissue level, the stress, strain, and SED distributions in the structures produced were determined, by calculation of the maximum principal stress and strain values and the SED values in each element.

\section{Results}

All models converged toward equilibrium solutions which resembled trabecular-like structures (Figures $3 a-c)$. The number of increments necessary to reach a stable solution was smaller for 


\section{Osteocyte model}

Surface cell model

Frequency (\% of bone tissue)

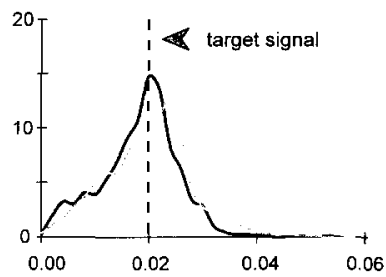

Strain energy density (MPa)

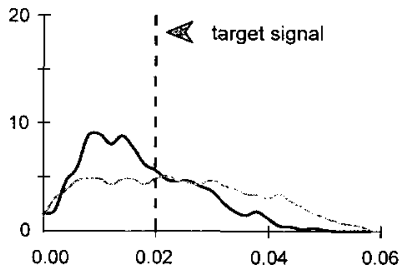

- load case 1

load case 2

Figure 4. The distributions are shown of strain energy density in the tissue of the equilibrium architectures produced by the osteocyte model (surface remodeling) and the surface cell model. The distributions for the osteocyte model are very similar for both load cases and peak at the value of the target signal. In contrast, no clear optimum is present in the distributions for the surface cell model.

"overall remodeling" than for "surface remodeling." In addition, the remodeling rate (although given in arbitrary units) was about two times higher for "overall remodeling" as compared to "surface remodeling." After convergence, the signals (SED) in the sensors averaged $0.02 \mathrm{MPa}$, which is equal to the value of the reference signal $k$, in all models.

The architectures produced by the osteocyte model described earlier and the osteocyte model, in which remodeling was only allowed at the bone-marrow interface, were not identical but very similar. The apparent properties of the equilibrium architectures were very similar as well. In these architectures, the axes of orthotropy were identical to the external loading directions. Furthermore, the proportion between the principal Young's moduli, $E_{11}$ and $E_{22}$ was between 1.7 and 2.0 (Table 1), which approximates the proportion between the load magnitudes $\sigma_{1}$ and $\sigma_{2}$. In other words, the anisotropy of these structures matched the externally applied loads. Both the relative apparent densities and the maximal principal Young's moduli were somewhat higher $(2-6 \%)$ in the structures produced by surface remodeling than those produced by overall remodeling (Table 1).

The surface cell model produced very different architectures compared to the osteocyte models (Figure 3c). The equilibrium architecture after 400 increments shows clearly that, using this remodeling algorithm, the initial architecture is more persistent in the eventual result. Local adaptation occurs, but the equilibrium architecture is closer to the initial one than those produced by the osteocyte models. The equilibrium architecture for the first load case was not orthotropic, which implies that the principal Young's moduli cannot be determined. Instead, the Young's moduli in the directions of the applied loads were determined. The apparent density in this architecture was about $11 \%$ higher than for the osteocyte model, whereas the apparent Young's modulus in the main loading direction was only $2 \%$ higher. The stiffnesses in all other directions were considerably higher than those in the osteocyte models. After changing the direction of loading, the trabeculae re-oriented. In this second architecture, which was rather similar to the initial configuration, both the apparent density and the apparent stiffness values were lower in the surface cell model than in the osteocyte models.

The evaluation of the mechanical variables at the tissue level revealed that, for the architectures produced by the osteocyte models, the SED values always varied around $0.02 \mathrm{MPa}$, which is equal to the target signal. In contrast, the SED distributions for the surface cell model have no clear optimum value (Figure 4).
Osteocyte model

Frequency ( $\%$ of bone tissue)

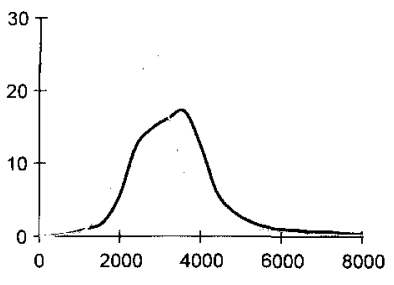

Maximal principal strain ( $\mu$ strain)

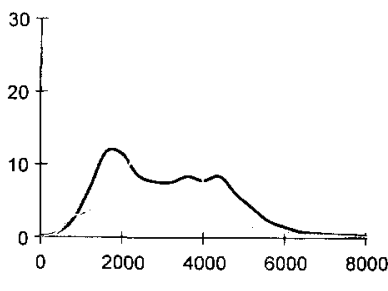

load case 2
Figure 5. The maximal principal strain values vary around $3000 \mu$ strain in the architectures produced by the osteocyte models. The maximal principal strain distributions for the surface cell model are different for each load case. In particular, the architecture produced by the first load case shows a much wider distribution, indicating that it is less well adapted to the applied loads.

With osteocytes as the sensors, the maximum principal stress and strain distributions were similar for the two load cases as well as for both the overall and surface remodeling algorithms. The narrow shape of the maximum principal strain distributions show that the greater part of the tissue was strained at a similar level (Figure 5). Compared to the osteocyte models, these distributions were much wider for the surface cell model and also varied more between the two equilibrium architectures.

\section{Discussion}

Previously, we have considered a strain adaptive bone-remodeling theory for which it was assumed that osteocytes appraise mechanical signals and regulate bone adaptation. ${ }^{14}$ Adaptation of bone density could take place at any location within the tissue represented in the model. It is known, however, that bone remodeling in trabecular bone occurs only at surfaces. A more realistic description of the process, based on surface remodeling, was compared with the previous model. Both remodeling algorithms produced qualitatively similar architectures. This is not surprising, because although the previous model allowed remodeling to take place throughout the tissue, it was in fact limited predominantly to the surfaces of existing trabeculae. ${ }^{14}$ Nevertheless, it was found that remodeling occurs much faster in overall remodeling as compared to surface remodeling. This is caused by the restricted volume available for simultaneous transformation in the latter case. We conclude, however, that the results from the surface remodeling algorithm are very similar to the ones from the overall remodeling algorithm reported earlier. ${ }^{14}$

The second goal of this project was to establish whether bone surface cells, such as lining cells and osteoblasts, could potentially regulate bone remodeling by themselves, without mechanosensory stimuli from osteocytes. For this purpose, two regulation models were compared. Some limitations of these models need to be discussed. First, trabecular bone tissue was represented in two dimensions. This limits comparison with actual bone, but presumably has no consequences for the comparison between the regulatory schemes, because van Rietbergen et al. ${ }^{20}$ showed that the osteocyte-based regulation process behaves similarly in a three-dimensional model. Second, the use of square elements limits the representation of trabecular geometry. This is partly resolved by allowing intermediate bone densities in boundary elements (Figure 2). More importantly however, digital models 
introduce solution errors at boundary elements. It has been shown that these errors can be significant and that further refinement of the element mesh does not necessarily reduce these boundary artifacts. ${ }^{7}$ The use of smooth boundary models does not solve this problem, for these were shown to produce similar errors. $^{5}$ To minimize boundary artifacts, the sensors in the surface cell model were chosen at the centers of the boundary elements (as opposed to the element nodes). Nevertheless, errors are inevitable when calculating signals in boundary elements and no solution for this problem is yet available. ${ }^{5}$ However, the effect of these errors on the remodeling results should be limited, because the pattern of boundary errors is oscillatory ${ }^{5}$ and therefore, errors are averaged out by the sensor influence function, which "filters" the erroneous oscillations and reduces their effect on the stimulus distribution. Another limitation is that only one specific load case was considered, whereas in reality bone is loaded by a variety of loading patterns, changing both in amplitude and direction. However, this probably does not affect the quality of the differences found between the models and it facilitates the assessment of effectiveness of the resulting architectures considerably. Furthermore, only net bone loss or gain was considered. Hence, the models do not account for separate effects of osteoblasts and osteoclasts and effects associated with the remodeling sequence. Finally, strain energy density was chosen as the mechanical signal, because the models used here are too coarse to obtain a more precise measure of the mechanical signals that osteocytes or lining cells perceive when the tissue is loaded. Strain energy density is a measure of the energy stored as a result of deformation of the tissue, and may be considered as a reasonable indication of the mechanical deformation which the cells experience. Although in fact, it is still unclear how bone cells sense mechanical signals, it is also uncertain how a more accurate representation of the mechanical signals might affect the results.

It was found that bone remodeling regulated by osteocytes is very effective indeed. The bone mass is distributed such that the apparent stiffness of the architecture is very well adapted to the externally applied loads, i.e., the equilibrium architecture has properties of (near) minimal mass for a certain average strain energy density value. As a result, the total mass of the structure produced is relatively independent of the loading direction. However, with surface cells as sensors, remodeling is less sensitive to the external loads, which is manifested by less change in the architecture. When bone remodeling is regulated by osteocytes, it leads primarily to adaptation of the architecture, but if it is regulated by surface cells, it mainly causes adaptation of strut thickness in the existing architecture, which leads to changes in the total mass. Apparently, with osteocytes as sensors, the remodeling process drifts more easily toward a significantly different morphology. Bone adaptation is less effective for changes of the loading directions with surface cells as sensors. This is evident from the frequency distribution plots, which indicate that parts of the structure receive relatively little loading, whereas other parts carry relatively high loads. It might be argued, however, that in reality loading patterns are relatively constant. For changes in load magnitudes only, surface cells should be equally capable to regulate adaptation as osteocytes, since changes in the load magnitudes require changes in mass rather than in architecture. It should be noted that adding surface cells to the osteocyte model makes no difference for the output of the model. This is due to the choice of the surface cell location in the boundary elements, which are also included in the tissue area where osteocytes are located.

Computational models, according to which strain derived signals are evaluated at the tissue surface and where bone mass is adapted accordingly, as in the present surface cell model, have been introduced by Luo et al. ${ }^{10}$ and Siffert et al. ${ }^{19}$ They found changes in strut thickness after changing the load magnitudes in a unit cell model, ${ }^{19}$ but also shape changes when applying loads to idealized structures. However, their results cannot be easily extrapolated to larger, more complex structures, which inhibits comparison with our results.

The present results indicate that osteocytes would be more efficient sensors than bone surface cells, in the sense that they produce architectures with a more appropriate mass distribution relative to the applied loads. It has long been suggested ${ }^{25}$ or even implicitly assumed that bone is an optimal structure, however, it is uncertain if this is in fact the case. Therefore, the superior performance of osteocytes as sensors gives no direct evidence that they actually fulfill this role.

In conclusion, the incorporation of surface remodeling into the remodeling algorithm had no essential effects on the architectures produced or their properties. The regulation of functional bone adaptation by mechano-sensitive osteocytes would be the most effective modality. This indicates that mechanical information at the bone surface may not be sufficient to adequately regulate functional bone adaptation.

\section{References}

1. Burger, E. H. and Veldhuijzen, J. P. Influence of mechanical factors on bone formation, resorption and growth in vitro. In: Hall, B. K., Ed. Bone. Vol. 7. Boca Raton: CRC; 1993; 37-56.

2. Cowin, S. C., Moss-Salentijn L., and Moss, M. L. Candidates for the mechanosensory system in bone. J Biomech Eng 113:191-197; 1991.

3. Currey, J. D. The effect of porosity and mineral content on the Young's modulus of elasticity of compact bone. J Biomech $21: 131-139 ; 1988$.

4. El-Haj, A. J., Minter, S. L. Rawlinson, S. C., Suswillo, R., and Lanyon, L. E. Cellular responses to mechanical loading in vitro. J Bone Miner Res 5:923$932 ; 1990$

5. Guldberg, R. E. and Hollister, S. J. Finite element solution errors associated with digital image-based mesh generation. Trans ASME, 1994 Adv Bioengin BED 28:147-148; 1994.

6. Harrigan, T. P. and Hamilton, J. J. Bone strain sensations via transmembrane potential changes in surface osteoblasts: loading rate and microstructural implications. J Biomech 26:183-200; 1993.

7. Hollister, S. J., Brennan, J. M., and Kikuchi, N. Homogenization sampling analysis of trabecular bone microstructural mechanics. Recent advances in computer methods in biomechanics and biomedical engineering. Swansea, UK: Books and Journals Int. Ltd.; 1992; 308-317.

8. Klein-Nulend J., Van der Plas, A., Semeins, C. M., Ajubi, N. E., Frangos, J. A., Nijweide, P. J., and Burger, E. H. Sensitivity of osteocytes to biomechanical stress in vitro. FASEB J 9:441-445; 1995.

9. Lanyon, L. E. Osteocytes, strain detection, bone modeling and remodeling. Calcif Tissue Int 53(Suppl. 1):S102-S106; 1993

10. Luo, G., Cowin, S. C., Sadegh, A. M., and Arroman, Y. P. Strain rate and strain as bone remodeling stimuli. In: Odgaard, A. and Weinans, H., Eds. Bone structure and remodeling, Singapore: World Scientific; $1995 ; 189-200$.

11. Marotti, G., Cané, V., Palazzini, S., and Palumbo, C. Structure-function relationships in the osteocyte. Ital J Miner Electrolyte Metab 4:93-106; 1990.

12. Miller, S. C., De Saint Georges, L., Bowman, B. M., and Jee, W. S. Bone lining cells: Structure and function. Scanning Microsc 3:953-960; 1989.

13. Mullender, M. G., Huiskes, R. and Weinans, H. A physiological approach to the simulation of bone remodeling as a self-organizational control process. J Biomechan 27:1389-1394; 1994.

14. Mullender, M. G. and Huiskes, R. A proposal for the regulatory mechanism of Wolff's law. J Orthop Res 13:503-512; 1995.

15. Mullender, M. G., Van der Meer, D. D., Huiskes, R., and Lips, P. Osteocyte density changes in aging and osteoporosis. Bone $18: 109-113 ; 1996$.

16. Mullender, M. G., Huiskes, R., Versleyen, H., and Buma, P. Osteocyte density and histomorphometric parameters in cancellous bone of the proximal femur in five mammalian species. J Orthop Res 14:972-979; 1996.

17. Parfitt, A. M. The cellular basis of bone remodeling: the quantum concept reexamined in the light of recent advances in the cell biology of bone. Calcif Tissue Int 36(Suppl 1):S37-45; 1984.

18. Skerry, T. M., Bitensky, L., Chayen, J., and Lanyon, L. E. Early strain-related 
changes in enzyme activity in osteocytes following bone loading in vivo. J Bone Min Res 4:783-788; 1989.

19. Siffert, R. S., Luo, G. M., Cowin, S. C., and Kaufman, J. J. Dynamic relationships of trabecular bone density, architecture and strength in a computational model of osteopenia. Bone 18:197-206; 1996.

20. Van Rietbergen B., Mullender M., and Huiskes R. Differentiation to plate-like or strut-like architectures in trabecular bone as a result of mechanical loading. Trans 41 th Annual Meeting Orthop Res Soc, Orlando, FL, Feb. 13-16, 1995; 179.

21. Van Rietbergen, B., Weinans, H., Huiskes, R., and Odgaard, A. A new method to determine trabecular bone elastic properties and loading using micromechanical Finite-Element models. J Biomech 28:69-81; 1995.

22. Van Rietbergen, B., Mullender, M. G., and Huiskes, R. A three dimensional model for the osteocyte-regulated remodeling simulation at the tissue level. In Middleton, J., Ed. Computer Methods in Biomechanics and Biomedical Engineering Books \& Journals International, Swansea, UK; 1996; 73-83.
23. Van Rietbergen, B., Odgaard, A., Kabel, J., and Huiskes, R. Direct mechanics assessment of mechanical symmetries and properties of trabecular bone architecture. J Biomech 29:1653-1657; 1996.

24. Weinbaum, S., Cowin, S. C., and Zeng, Y. A model for the excitation of osteocytes by mechanical loading-induced bone fluid shear stresses. J Biomechan 27:339-360; 1993.

25. Wolff, J. Das Gesetz der Transformation der Knochen. Berlin: A. Hirchwild: 1892. The law of bone remodeling. Translated by Maquet. $P$. and Furlong, $R$. Berlin: Springer; 1986.
Date Received: December 18. 1996 Date Revised: February 25, 1997 Date Accepted: March 3, 1997 